\title{
Assessment of outcomes and novel immune biomarkers in metaplastic breast cancer: a single institution retrospective study
}

Evan Morgan ${ }^{1,2}$, Anupama Suresh ${ }^{1,2}$, Akaansha Ganjü $^{3}$, Daniel G. Stover ${ }^{1,2}$, Robert Wesolowski ${ }^{1,2}$, Sagar Sardesai ${ }^{1,2}$, Anne Noonan ${ }^{1,2}$, Raquel Reinbolt ${ }^{1,2}$, Jeffrey VanDeusen ${ }^{1,2}$, Nicole Williams ${ }^{1,2}$, Mathew A. Cherian ${ }^{1,2}$, Zaibo Li ${ }^{4}$, Gregory Young ${ }^{5}$, Marilly Palettas ${ }^{5}$, Julie Stephens ${ }^{5}$, Joseph Liu ${ }^{1,2}$, Amanda Luff ${ }^{1,2}$, Bhuvaneswari Ramaswamy ${ }^{1,2}$ and Maryam Lustberg ${ }^{1,2^{*}}$

\begin{abstract}
Background: Metaplastic breast cancer remains poorly characterized given its rarity and heterogeneity. The majority of metaplastic breast cancers demonstrate a phenotype of triple-negative breast cancer; however, differences in clinical outcomes between metaplastic breast cancer and triple-negative breast cancer in the era of third-generation chemotherapy remain unclear.
\end{abstract}

Methods: We compared the clinical outcomes between women with metaplastic breast cancer and women with triple-negative breast cancer diagnosed between 1994 and 2014. Metaplastic breast cancer patients were matched 1:3 to triple-negative breast cancer patients by stage and age at diagnosis. Distant disease-free survival (DDFS) and overall survival (OS) were estimated using Kaplan Meier methods and Cox proportional hazard regression models. Immune checkpoint markers were characterized by immunohistochemistry in a subset of samples.

Results: Forty-four metaplastic breast cancer patients (stage I 14\%; stage II 73\%; stage III 11\%; stage IV 2\%) with an average age of $55.4( \pm 13.9)$ years at diagnosis. Median follow-up for the included metaplastic breast cancer and triple-negative breast cancer patients $(n=174)$ was $2.8(0.1-19.0)$ years. The DDFS and OS between matched metaplastic breast cancer and triple-negative breast cancer patients were similar, even when adjusting for clinical covariates (DDFS: $\mathrm{HR}=1.64, p=0.22 ; \mathrm{OS}: \mathrm{HR}=1.64, p=0.26$ ). Metaplastic breast cancer samples $(n=27)$ demonstrated greater amount of CD163 in the stroma $(p=0.05)$ and PD-L1 in the tumor $(p=0.01)$ than triplenegative breast cancer samples $(n=119)$, although more triple-negative breast cancer samples were positive for CD8 in the tumor than metaplastic breast cancer samples ( $p=0.02)$.

Conclusions: Patients with metaplastic breast cancer had similar outcomes to those with triple-negative breast cancer based on DDFS and OS. The immune checkpoint marker profile of metaplastic breast cancers in this study may prove useful in future studies attempting to demonstrate an association between immune profile and survival.

Keywords: Metaplastic breast cancer, Clinical outcomes, Distant disease-free survival, Overall survival, Triple-negative breast cancer, Immune markers

\footnotetext{
* Correspondence: Maryam.Lustberg@osumc.edu

'Stefanie Spielman Comprehensive Breast Cancer, The Ohio State University,

Columbus, OH, USA

${ }^{2}$ Division of Medical Oncology, Comprehensive Cancer Center, The Ohio

State University Medical Center, Columbus, OH, USA

Full list of author information is available at the end of the article
}

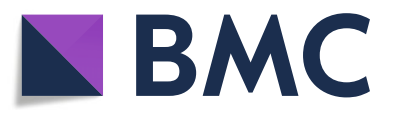

(c) The Author(s). 2020 Open Access This article is distributed under the terms of the Creative Commons Attribution 4.0 International License (http://creativecommons.org/licenses/by/4.0/), which permits unrestricted use, distribution, and reproduction in any medium, provided you give appropriate credit to the original author(s) and the source, provide a link to the Creative Commons license, and indicate if changes were made. The Creative Commons Public Domain Dedication waiver (http://creativecommons.org/publicdomain/zero/1.0/) applies to the data made available in this article, unless otherwise stated. 


\section{Introduction}

Metaplastic breast cancer (MBC) is a very rare type of invasive breast cancer in which the original cell type, usually glandular epithelium, differentiates into either epithelial and/or mesenchymal cell types with glandular and nonglandular components [1, 2]. According to the World Health Organization (WHO), MBC includes several subtypes, including low-grade adenosquamous carcinoma, fibromatosis-like metaplastic carcinoma, squamous cell carcinoma, spindle cell carcinoma, mixed metaplastic carcinoma, myoepithelial carcinoma, and metaplastic carcinoma with mesenchymal differentiation-notably chondroid, osseous, and other types of mesenchymal differentiation [3]. MBC accounts for $0.2-5 \%$ of all breast cancers, yet the lack of an accepted definition may contribute to the varying prevalence rates [4].

The majority of MBCs are triple-negative, which is defined as breast tumors that are negative for the estrogen receptor, progesterone receptor, and do not overexpress HER2/neu. Furthermore, an immunohistochemical panel showed that $93.8 \%$ of $\mathrm{MBCs}$ were basal-like, the most common subset of triple-negative breast cancer (TNBC) [5]. MBCs tend to have a large tumor size, rapid growth, and less axillary lymph node involvement [6, 7]. Although there is less axillary node involvement, like soft tissue sarcomas, MBCs are more prone to hematogenous dissemination and have a poor prognosis [8, 9]. While this may be in part due to the preponderance of the TNBC phenotype among MBCs, one prior study comparing $\mathrm{MBC}$ and triple-negative invasive ductal cancers showed that MBCs had worse prognosis relative to TNBCs, with shorter disease-free survival (DFS) in patients with nodal metastasis treated with adjuvant chemotherapy [10].

Patients with $\mathrm{MBC}$ are significantly more likely to receive chemotherapy compared to patients with invasive carcinoma of no special type (IC-NST) [11], the most common histologic type of breast cancer. However, $\mathrm{MBC}$ has demonstrated resistance to traditional forms of chemotherapy $[9,12]$. It is important to assess how the treatment of these cancers has evolved over time, given disparities in clinical outcomes between $\mathrm{MBC}$ and other TNBC. Notably, the rarity of MBC leads to difficulty in identifying effective treatment strategies through clinical trials. Although evidence from randomized controlled trials supporting the use of neoadjuvant or adjuvant chemotherapy for $\mathrm{MBC}$ is limited, the majority of patients with $\mathrm{MBC}$ receive chemotherapy, given the high risk of relapse and poor prognosis [13].

In recent years, immunotherapeutic agents that target components of tumor microenvironments (TMEs) have shown potential for the treatment of TNBC; however, clinical trials are still ongoing[14-16]. The interaction between the immune regulatory proteins, programmed cell death-1 (PD-1) and programmed death ligand-1 (PD-L1), is of particular interest as it has been shown to facilitate tumor progression through inactivation of tumor-infiltrating lymphocytes (TILs) [17, 18]. High levels of the immune regulatory protein $\mathrm{PD}-\mathrm{L} 1$ are seen in both TNBC and MBC, though PD-L1 expression is higher in MBCs $[19,20]$. Clinical trials utilizing PD-L1 and PD-1 inhibitors in the treatment of TNBC are ongoing, with results demonstrating response to immunotherapy as first-line therapy or in combination with chemotherapy [21-24]. Infiltration of cytotoxic $\mathrm{T}$ cells $\left(\mathrm{CD}^{+} \mathrm{T}\right.$ cells $)$, which are negatively regulated by $\mathrm{PD}-1$, in residual tumors is associated with better clinical outcomes in TNBC treated with neoadjuvant chemotherapy [25], though this relationship has not yet been examined in MBC. CD163, a scavenger receptor for the hemoglobin-haptoglobin complex, is a marker for alternatively activated (M2) polarized macrophages [26]. CD $163^{+}$tumor-associated macrophage (TAM) infiltration in tumor stroma is also of clinical interest as it is strongly associated with TNBC and is associated with poorer survival in TNBC with low TIL levels [27-29], though the role of CD163 ${ }^{+}$ TAM infiltration in MBC has not been examined.

Given its rare occurrence and heterogeneous classification, MBC remains poorly characterized. While most $\mathrm{MBC}$ is triple-negative and treated as such, the differences in outcomes between MBC and TNBC led us to perform a 1:3 matched comparison to evaluate survival outcomes in the era of third-generation chemotherapy. In this retrospective study, we aimed to compare distant disease-free survival (DDFS) and overall survival (OS) between patients with MBC treated at The Ohio State University Comprehensive Cancer Center (OSUCCCJames) and those with non-metaplastic TNBC. Further, we assessed and compared immune marker expression in the TME of MBC and TNBC with a goal to identify immune markers which can be potential targets and provide prognostic value.

\section{Methods \\ Study design}

Patients treated at The Ohio State University Comprehensive Cancer Center-Arthur G. James Cancer Hospital and Richard J. Solove Research Institute between January 1, 1994, and December 31, 2014, with a diagnosis of TNBC or MBC were eligible for this retrospective study. Following IRB approval (OSU 2015C0135), the list of patients fulfilling this eligibility criterion, i.e., any woman with a diagnosis of TNBC or MBC between January 1, 1994, and December 31, 2014, was obtained from Ohio State University Wexner Medical Center and James Cancer Registry. Patients with ICD-O-3 Histology Code associated with "Metaplastic carcinoma, NOS" were identified through the OSUCCC-James Cancer 
Registry, and $\mathrm{MBC}$ diagnosis was confirmed through pathology report review prior to inclusion in the study. Patients with ICD 9 code diagnosis of breast cancer (174.0-174.9) and pathology negative for estrogen, progesterone and HER2 receptor overexpression were considered to have TNBC. The OSUCCC-James Cancer Registry determined receptor status through the review of individual pathology reports utilizing the guidelines from the College of American Pathologists (CAP) and the American Society of Clinical Oncology (ASCO) that were available at the time of diagnosis. Patient charts with incomplete data were excluded from the study. Each MBC patient was matched with three nonmetaplastic TNBC patients based on stage and age at diagnosis [30]. Stage (I-IV) was required to be identical and age at diagnosis was restricted to within 10 years.

\section{Data collection}

The following data were extracted from patients' medical records: patient's age at time of diagnosis, race, ethnicity, height, weight, stage, biomarker profiles (ER, PR, and HER2) of the tumor, therapy modality (surgery, chemotherapy type and regimen, and radiotherapy), and duration, as well as survival data including distant diseasefree survival and overall survival.

\section{Multi-color multiplex immunohistochemistry and assessment of checkpoint immune system}

Multi-color multiplex immunohistochemical (IHC) assays capable of demonstrating co-localization of PD-L1 with CD8 and CD163 were performed on fresh-cut whole sections from patients' resection specimens on an Autostainer BenchMark XT platform (Ventana Medical Systems, Inc., Tucson, AZ; Ventana) according to the manufacturer's recommendation. The antibodies used were as follows: for PD-L1 clone SP263, rabbit, Ventana; for CD8 clone SP57, rabbit, Ventana; for CD163 clone MRQ26, mouse, Ventana. SP263 is the only available PD-L1 antibody in the multiplex IHC assay. PD-L1 signal was detected using iVIEW DAB IHC Detection Kit (Ventana) with brown color, CD8 signal was detected using iVIEW HRP Green IHC Detection Kit (Ventana) with green color and CD163 signal was detected using iVIEW Fast Red IHC Detection Kit (Ventana) with red color. IHCs were evaluated by a pathologist (ZL) using a semi-quantitative approach for the entire tumor and tumor-surrounding areas under a microscope. Positive (previously confirmed PD-L1-positive breast carcinoma specimen) and negative (previously confirmed PD-L1negative breast carcinoma specimen) controls were included for each batch of IHCs. Membranous PD-L1 staining in tumor cells or immune cells was considered as a specific staining. A positive PD-L1 expression among tumor cells was defined as any membranous staining in $\geq 1 \%$ of tumor cells in order to maximize the assay sensitivity for PD-L1-positive cases [31, 32]. The following parameters were assessed: PD-L1 expression in tumor cells, PD-L1 expression in immune cells, CD8+ immune cells within the tumor, CD8+ immune cells within the stroma, CD163+ macrophages within the tumor, and CD163+ macrophages within the stroma. The cut-off percentage for CD8+ cells and CD163+ cells was set at 10\% [32].

\section{Statistical analysis}

Analyses focused on the comparisons between the age at initial diagnosis and stage-matched MBC and TNBC patients. The primary endpoints of this study were DDFS and OS. The DDFS period was defined as the time from diagnosis to the date of the first observation of distant disease recurrence, while the OS period was defined as the time from diagnosis to death or censoring. An exploratory endpoint was to define the expression of immune markers in the TME of samples from primary $\mathrm{MBC}$. A comparison of clinicopathological and treatment characteristics was achieved using a two-sample $t$ test for continuous variables and a Fisher's exact test for categorical variables. Kaplan-Meier methods were used to estimate survival curves for OS and DDFS for the two groups. Patients diagnosed as stage IV were eliminated from analyses of DDFS. The log-rank test was used to compare the curves. Cox proportional hazard regression models were used to examine DDFS and OS between $\mathrm{MBC}$ and TNBC groups while adjusting for additional relevant clinical covariates such as age at initial diagnosis, stage, use of chemotherapy and radiotherapy, and lymph node involvement.

\section{Results}

\section{Patient characteristics}

Our review of medical records identified 382 patients who were eligible for this study. Of these, 44 had MBC while the remaining 338 patients had non-metaplastic TNBC. Each MBC patient was matched with three TNBC patients based on age and stage except for one 93-year-old MBC patient who had only one age- and stage-matched TNBC patient. Hence, 130 TNBC patients were included in the study. Median follow-up, defined as the time from diagnosis to death or censoring, for the included MBC and TNBC patients $(n=174)$ was $2.8(0.1-19.0)$ years, with only 8 patients followed for more than 10 years.

The demographic data of this population are listed in Table 1. The average age for MBC patients was $55.4( \pm 13.9)$ years at diagnosis. The majority of patients with MBC presented as stage II breast cancer (72.7\%). Fewer MBC patients were node-positive at the presentation when compared to TNBC, though the difference was not significant $(29.5 \%$ vs $46.2 \%, p=0.08)$. 
Table 1 Demographic and clinical data by group

\begin{tabular}{|c|c|c|c|c|c|}
\hline Variable & Level & $\operatorname{TNBC}(n=130)$ & $\operatorname{MBC}(n=44)$ & Total & $p$ value \\
\hline Age at initial diagnosis & Mean (SD) & $54.6(12.8)$ & $55.4(13.9)$ & $54.8(13.0)[n=174]$ & 0.7495 \\
\hline \multirow[t]{3}{*}{ Race } & White & $108(83.1 \%)$ & $40(90.9 \%)$ & $148(85.1 \%)$ & 0.2451 \\
\hline & Black & $14(10.8 \%)$ & $4(9.1 \%)$ & $18(10.3 \%)$ & - \\
\hline & Other & $8(6.2 \%)$ & $0(0.0 \%)$ & $8(4.6 \%)$ & - \\
\hline Hispanic ethnicity & Yes & $3(2.3 \%)$ & $0(0.0 \%)$ & $3(1.8 \%)$ & 1.0000 \\
\hline Positive nodes & Yes & $60(46.2 \%)$ & $13(29.5 \%)$ & $73(42.0 \%)$ & 0.0765 \\
\hline \multirow[t]{4}{*}{ Stage } & । & $16(12.3 \%)$ & $6(13.6 \%)$ & $22(12.6 \%)$ & 0.9812 \\
\hline & $\|$ & $96(73.8 \%)$ & $32(72.7 \%)$ & $128(73.6 \%)$ & - \\
\hline & III & $15(11.5 \%)$ & $5(11.4 \%)$ & $20(11.5 \%)$ & - \\
\hline & IV & $3(2.3 \%)$ & $1(2.3 \%)$ & $4(2.3 \%)$ & - \\
\hline \multirow[t]{2}{*}{ ER status } & Positive & $0(0.0 \%)$ & $4(9.1 \%)$ & $4(2.3 \%)$ & 0.0037 \\
\hline & Negative & $130(100.0 \%)$ & 40 (90.9\%) & $170(97.7 \%)$ & - \\
\hline \multirow[t]{2}{*}{ PR status } & Positive & $0(0.0 \%)$ & $4(9.1 \%)$ & $4(2.3 \%)$ & 0.0037 \\
\hline & Negative & $130(100.0 \%)$ & $40(90.9 \%)$ & 170 (97.7\%) & - \\
\hline \multirow[t]{2}{*}{ HER2 status } & Positive & $0(0.0 \%)$ & $1(2.3 \%)$ & $1(0.6 \%)$ & 0.0146 \\
\hline & Negative & $130(100.0 \%)$ & 43 (97.7\%) & 170 (98.3\%) & - \\
\hline
\end{tabular}

$p$ values from a two-sample $t$ test for continuous variables and a Fisher's exact test for categorical variables. Abbreviations: $E R=$ estrogen receptor; $H E R 2=$ human epidermal growth factor receptor $2 ; M B C=$ metaplastic breast cancer; $P R=$ progesterone receptor; $S D=$ standard deviation; $T N B C=$ triple-negative breast cancer

\section{Treatment}

The details of the treatment modalities are summarized in Table 2. Overall treatment modalities were similar, including rates of type of surgery (lumpectomy versus mastectomy), radiation, and receipt of any type of chemotherapy. Among specific agents, taxanes were used less frequently for the treatment of $\mathrm{MBC}$ patients compared to non-metaplastic TNBC patients $(70.5 \%$ vs $85.4 \%, p=0.0411)$. Among MBC patients, there were three patients with estrogen receptor (ER)-positive and progesterone receptor (PR)-negative (ER+/PR-) MBC ( $\leq$ $10 \%$ cells $\mathrm{ER}+)$, three patients with ER-negative and PRpositive $(\mathrm{ER}-/ \mathrm{PR}+) \mathrm{MBC}(\leq 10 \%$ cells $\mathrm{PR}+)$, and one patient with $\mathrm{ER}+/ \mathrm{PR}+\mathrm{MBC}(<10 \%$ cells $\mathrm{ER}+/ \mathrm{PR}+)$. Two patients with $E R+/ P R-M B C$ received a form of antiestrogen therapy, while the third had a previous history of bilateral oophorectomy. Two patients with ER-/PR+ $M B C$ received a form of anti-estrogen therapy, while the third patient did not. One patient with HER2 positive (HER2+) MBC received anti-HER2 therapy.

Table 2 Treatment data by group

\begin{tabular}{|c|c|c|c|c|c|}
\hline Variable & Level & $\operatorname{TNBC}(n=130)$ & $\mathrm{MBC}(n=44)$ & Total & $p$ value \\
\hline \multirow[t]{6}{*}{ Local therapy } & None & $4(3.1 \%)$ & $1(2.3 \%)$ & $5(2.9 \%)$ & 0.8203 \\
\hline & Complete mastectomy & $33(25.4 \%)$ & $15(34.1 \%)$ & $48(27.6 \%)$ & - \\
\hline & Lumpectomy & $2(1.5 \%)$ & $1(2.3 \%)$ & $3(1.7 \%)$ & - \\
\hline & Radiation therapy & $2(1.5 \%)$ & $0(0.0 \%)$ & $2(1.1 \%)$ & - \\
\hline & Complete mastectomy + radiation therapy & $37(28.5 \%)$ & $13(29.5 \%)$ & $50(28.7 \%)$ & - \\
\hline & Lumpectomy + radiation therapy & $52(40.0 \%)$ & $14(31.8 \%)$ & $66(37.9 \%)$ & - \\
\hline Radiation & Yes & $91(70.0 \%)$ & $27(61.4 \%)$ & $118(67.8 \%)$ & 0.3509 \\
\hline Breast conserving surgery & Yes & $54(41.5 \%)$ & $15(34.1 \%)$ & $69(39.7 \%)$ & 0.4763 \\
\hline Chemotherapy in first year following diagnosis & Yes & $119(91.5 \%)$ & $38(86.4 \%)$ & $157(90.2 \%)$ & 0.3782 \\
\hline Anthracycline therapy & Yes & $104(80.0 \%)$ & $34(77.3 \%)$ & $138(79.3 \%)$ & 0.6731 \\
\hline Platinum therapy & Yes & $17(13.1 \%)$ & $6(13.6 \%)$ & $23(13.2 \%)$ & 1.0000 \\
\hline Taxane therapy & Yes & $111(85.4 \%)$ & $31(70.5 \%)$ & $142(81.6 \%)$ & 0.0411 \\
\hline
\end{tabular}

Chemotherapy regimens in which at least 5 patients received were included. $p$ values from a two-sample $t$ test for continuous variables and a Fisher's exact test for categorical variables. Abbreviations: $M B C=$ metaplastic breast cancer, $T N B C=$ triple-negative breast cancer 


\section{Clinical outcomes}

The median DDFS for MBC patients was 10.9 years vs 13.7 years for non-metaplastic TNBC patients. We did not detect a statistically significant difference in 3- and 5-year DDFS between the two groups with the DDFS for MBC patients of $77.5 \%$ and $77.5 \%$ vs $81.7 \%$ and $78.7 \%$ ( $\log$ rank $p=0.35$; Fig. 1) for non-metaplastic TNBC patients, respectively. There were too few deaths among the non-metaplastic patients to reach the median overall survival in the TNBC group for OS. We did not detect a statistically significant difference in OS between the groups (log-rank $p=0.32$, Fig. 2). Estimates of 3- and 5year OS were $78.9 \%$ and $78.9 \%$.vs $86.1 \%$ and $81.4 \%$ for $\mathrm{MBC}$ and TNBC patients, respectively.

Multivariate analyses (Table 3) showed that there was no significant difference in DDFS $(\mathrm{HR}=1.64$, $95 \% \mathrm{CI}=0.75-3.58, p=0.22)$ and $\mathrm{OS}(\mathrm{HR}=1.64$, 95\% CI $=0.69-3.90, p=0.26)$ for $\mathrm{MBC}$ vs TNBC patients, when adjusting for clinical stage, nodal status, age, use of any chemotherapy, and radiotherapy. Patients with nodal metastases showed decreased likelihood of DDFS $(\mathrm{HR}=4.32,95 \% \mathrm{CI}=1.66-11.29, p$ $=0.003)$ and a trend towards worse OS $(\mathrm{HR}=2.62$, $95 \% \mathrm{CI}=0.88-7.82, p=0.08)$. Stage was a significant predictor of DDFS $(p=0.03)$ with stage I $(\mathrm{HR}=0.33$, $95 \% \mathrm{CI}=0.06-1.83)$ and $\mathrm{II}(\mathrm{HR}=0.32,95 \% \mathrm{CI}=$ 0.14-0.74) patients having longer survival than stage III. Results for OS were similar $(p=0.002)$ with stage I $(\mathrm{HR}=0.06,95 \% \mathrm{CI}=0.01-0.54)$ and II $(\mathrm{HR}=0.07$,
95\% CI $=0.02-0.28)$ patients showing greater survival. No other variables had a significant effect on DDFS or OS following multivariate analysis.

Given differences in taxane use, we examined the use of taxane chemotherapies via an additional multivariate analysis using a categorical variable (no chemotherapy, taxane, and non-taxane) while adjusting for age, use of radiation therapy, nodal status, and metaplastic diagnosis. A majority (90\%, $n=142)$ of the patients who received chemotherapy received a taxane, and only a few patients $(\mathrm{n}=15)$ received non-taxane therapy. We observed no significant effect on DDFS $(p=0.56)$ or OS $(p=0.80)$ between taxane and non-taxane use.

\section{Immune checkpoint marker expression}

The expression of various immune markers within MBC is summarized in Table 4. PD-L1 expression on tumor cells was detected in $29.6 \%$ of MBC samples $(n=27)$. Immune marker testing was not possible for $38.6 \%$ of cases $(n=17)$ lacking sufficient tissue for staining (blocks or unstained slides). A separate cohort of TNBC patients $(n=119)$ was used to compare tissue immune markers with $\mathrm{MBC}$ samples, seen in Table 4. More MBC samples demonstrated $\mathrm{CD}_{163^{+}}$cells in the stroma ( $96.3 \%$ vs. $79.8 \%, p=0.0468$ ) and positive PD-L1 expression in tumor cells $(29.6 \%$ vs. $10.1 \%, p=0.0133)$ compared to TNBC samples. However, significantly more TNBC samples demonstrated high levels of $\mathrm{CD}^{+}$immune cells in the tumor compared to MBC samples ( $44.5 \%$ vs.

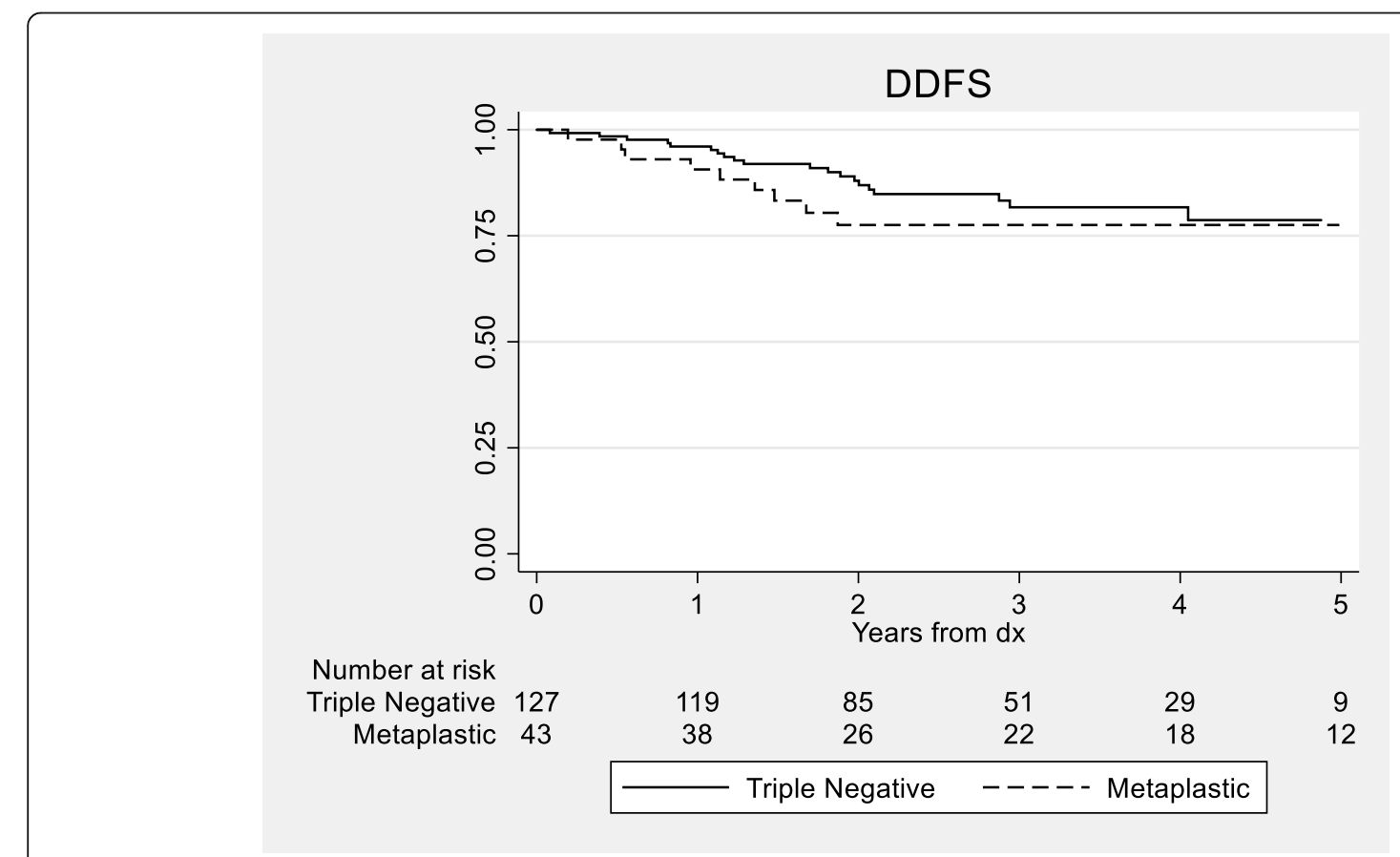

Fig. 1 Kaplan-Meier curves for distant disease-free survival between patients with metaplastic and triple-negative breast cancer $(p=0.35)$. Only the first 5 years of survival time is displayed, as few patients $(n=21)$ had follow-up past 5 years. Abbreviations: DDFS = distant disease-free survival; $d x=$ diagnosis 


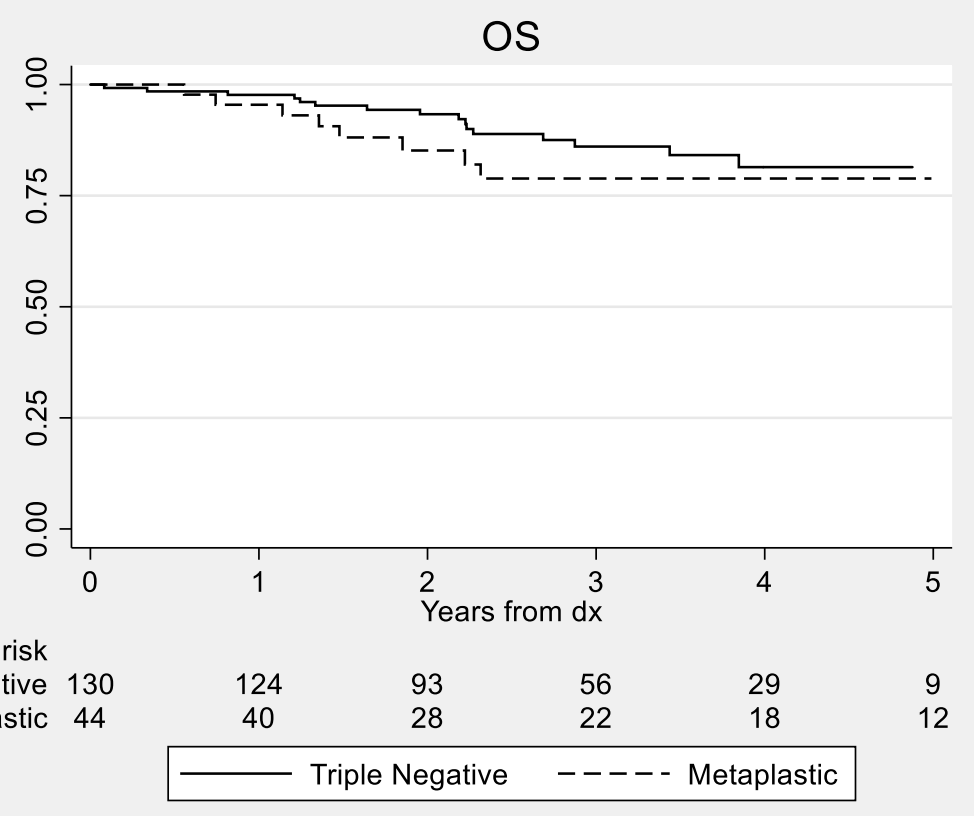

Fig. 2 Kaplan-Meier curves for overall survival between patients with metaplastic and triple-negative breast cancer $(p=0.32)$. Only the first 5 years of survival time is displayed, as few patients $(n=21)$ had follow-up past 5 years. Abbreviations: OS = overall survival; $d x=$ diagnosis

$18.5 \%, p=0.0158)$. Figure 3 shows images of the different immune markers and PD-L1 expressions in two metaplastic breast carcinomas. The immune marker profile of our MBC samples is analogous to those generated from TNBC samples in previous studies, which demonstrated associations between lower CD8, higher CD163, higher PD-L1 staining and worse prognosis $[25,28]$.

\section{Discussion}

$\mathrm{MBC}$ is a rare and heterogeneous type of invasive breast cancer that subsequently lacks research demonstrating consistent direction. Moreover, $\mathrm{MBC}$ is historically known to have an aggressive nature with a questionable response the chemotherapy. Our single institution, retrospective study compared the clinical and histopathologic features, management, outcomes, and immune marker expression between patients with $\mathrm{MBC}$ and a matched cohort of patients with TNBC. We found that patients with MBC had similar outcomes to TNBC based on DDFS and OS, unlike previous publications [6, 10, 33]. Furthermore, the study identified that treatment with a taxane or anthracycline type chemotherapy was common among our patients with MBC $(70.5 \%$ and $77.3 \%$ ), in contrast with rates seen in a previous report

Table 3 Multivariate Cox model for DDFS $(n=170)$ and OS $(n=174)$

\begin{tabular}{|c|c|c|c|c|}
\hline \multirow[t]{2}{*}{ Outcome } & \multicolumn{2}{|c|}{ MV analysis of DDFS with nodal status } & \multicolumn{2}{|c|}{ MV analysis of OS with nodal status } \\
\hline & $\mathrm{HR}(95 \% \mathrm{Cl})$ & $p$ value & HR $(95 \% \mathrm{Cl})$ & $p$ value \\
\hline Age at initial diagnosis & $1.01(0.98,1.04)$ & 0.6608 & $1.03(0.99,1.07)$ & 0.1252 \\
\hline $\begin{array}{l}\text { Chemotherapy given in the first } \\
\text { year following diagnosis }\end{array}$ & $0.42(0.11,1.65)$ & 0.2129 & $0.71(0.16,3.21)$ & 0.6552 \\
\hline Metaplastic diagnosis & $1.64(0.75,3.58)$ & 0.2159 & $1.64(0.69,3.90)$ & 0.2597 \\
\hline Positive nodes & $4.32(1.66,11.29)$ & 0.0028 & $2.62(0.88,7.82)$ & 0.0844 \\
\hline Radiotherapy given & $0.58(0.23,1.50)$ & 0.2643 & $0.55(0.20,1.49)$ & 0.2384 \\
\hline Stage 1 & $0.33(0.06,1.83)$ & 0.0287 & $0.06(0.01,0.54)$ & 0.0021 \\
\hline Stage 2 & $0.32(0.14,0.74)$ & - & $0.07(0.02,0.28)$ & - \\
\hline Stage 3 & Ref & - & $0.24(0.06,0.93)$ & - \\
\hline Stage 4 & - & - & Ref & - \\
\hline
\end{tabular}

Abbreviations: $\mathrm{Cl}=$ confidence interval; DDFS = distant disease-free survival; $H R=$ hazard ratio; $M V=$ multivariate; $O S=$ overall survival 
Table 4 Comparison of immune marker expression in TNBC and MBC samples

\begin{tabular}{|c|c|c|c|c|c|}
\hline \multicolumn{6}{|c|}{ Metaplastic breast carcinoma immune checkpoint markers } \\
\hline Outcome & Level & TNBC $(n=119)$ & $\mathrm{MBC}(n=27)$ & Total & $p$ value \\
\hline Age at initial diagnosis & Mean (SD) & $51.9(11.8)$ & $57.8(15.3)$ & $53.0(12.7)$ & 0.0281 \\
\hline \multirow[t]{2}{*}{$\mathrm{CD} 8 \%$ in stroma $\geq 10$} & No & $25(21.0 \%)$ & $6(22.2 \%)$ & $31(21.2 \%)$ & 1.0000 \\
\hline & Yes & $94(79.0 \%)$ & $21(77.8 \%)$ & $115(78.8 \%)$ & 0.2451 \\
\hline \multirow[t]{2}{*}{$\mathrm{CD}^{+} \%$ in tumor $\geq 10$} & No & $66(55.5 \%)$ & $22(81.5 \%)$ & $88(60.3 \%)$ & 0.0158 \\
\hline & Yes & $53(44.5 \%)$ & $5(18.5 \%)$ & $58(39.7 \%)$ & - \\
\hline \multirow[t]{2}{*}{$\mathrm{CD}_{163^{+}} \%$ in tumor $\geq 10$} & No & $64(53.8 \%)$ & $14(51.9 \%)$ & $78(53.4 \%)$ & 1.0000 \\
\hline & Yes & $55(46.2 \%)$ & $13(48.1 \%)$ & $68(46.6 \%)$ & 0.0765 \\
\hline \multirow[t]{2}{*}{$\mathrm{CD} 163^{+} \%$ in stroma $\geq 10$} & No & $24(20.2 \%)$ & $1(3.7 \%)$ & $25(17.1 \%)$ & 0.0468 \\
\hline & Yes & $95(79.8 \%)$ & $26(96.3 \%)$ & $121(82.9 \%)$ & - \\
\hline \multirow[t]{2}{*}{ PD-L1\% in tumor $\geq 1$} & No & 107 (89.9\%) & 19 (70.4\%) & $126(86.3 \%)$ & 0.0133 \\
\hline & Yes & $12(10.1 \%)$ & $8(29.6 \%)$ & $20(13.7 \%)$ & - \\
\hline \multirow[t]{2}{*}{ PD-L1\% in stroma $\geq 1$} & No & $32(26.9 \%)$ & $11(40.7 \%)$ & $43(29.5 \%)$ & 0.1665 \\
\hline & Yes & 87 (73.1\%) & $16(59.3 \%)$ & $103(70.5 \%)$ & - \\
\hline \multirow[t]{2}{*}{ PD-L1\% overall $\geq 1$} & No & $32(26.9 \%)$ & $12(44.4 \%)$ & $44(30.1 \%)$ & 0.1025 \\
\hline & Yes & 87 (73.1\%) & 15 (55.6\%) & 102 (69.9\%) & - \\
\hline
\end{tabular}

TNBC samples were obtained from a different cohort of patients. $p$ values from a two-sample t-test for continuous variables and a Fisher's exact test for categorical variables. Abbreviations: $M B C=$ metaplastic breast cancer; $S D=$ standard deviation; $T N B C=$ triple-negative breast cancer

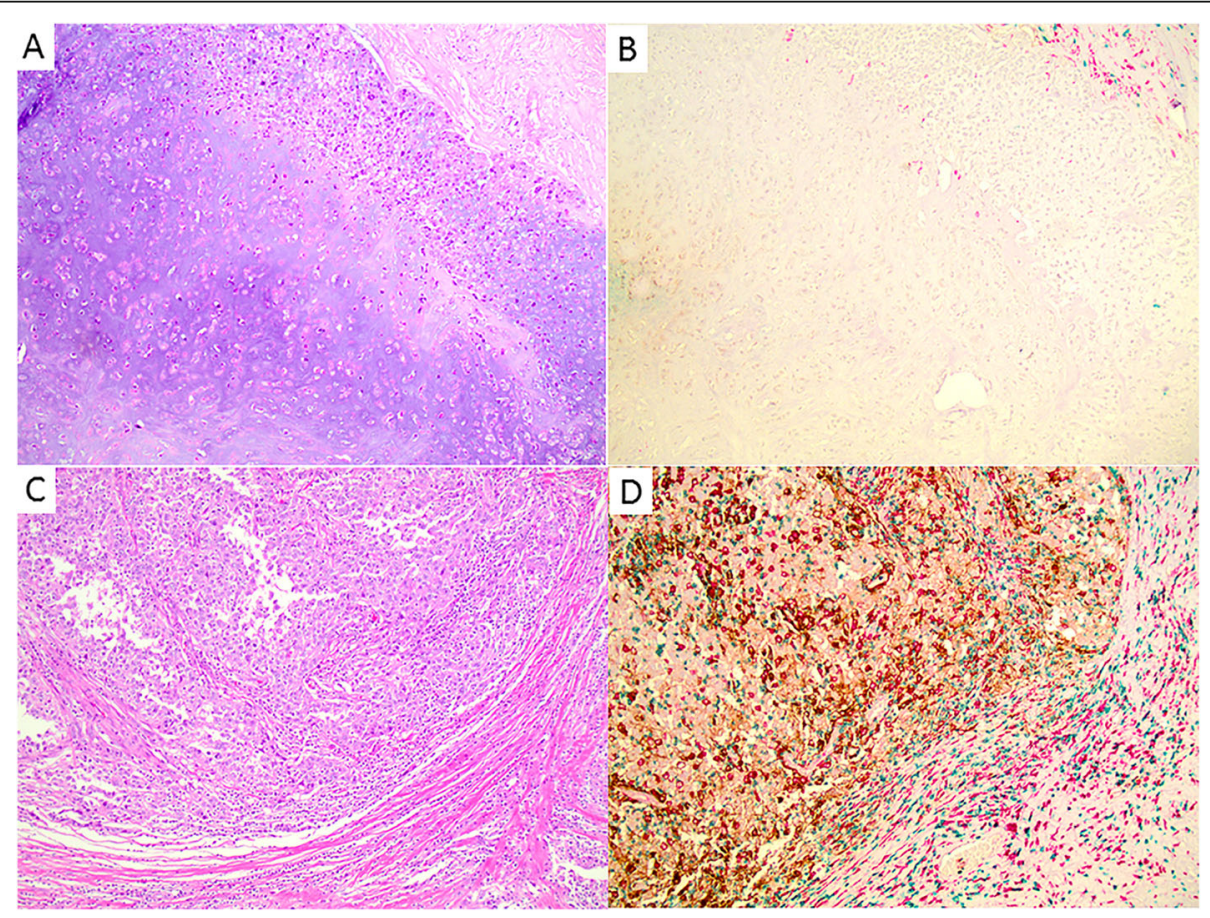

Fig. 3 Representative images of different immune reaction and PD-L1 expressions in two invasive metaplastic breast carcinomas, as detected with anti-PD-L1 multiplex immunohistochemistry (anti-CD8 in green, anti-CD163 in red, and anti-PD-L1 in brown). a, b One invasive metaplastic carcinoma with no PD-L1 expression, only scattered CD $163^{+}$cells and very rare CD8 ${ }^{+}$cytotoxic T-cells in the peritumoral stroma. $\mathbf{c}$, $\mathbf{d}$ One invasive metaplastic carcinoma with strong PD-L1 expression in tumor cells and stromal cells, diffuse CD163 ${ }^{+}$cells and CD8 $8^{+}$cytotoxic T-cells in tumoral stroma and peritumoral stroma. Magnification: $\times 100$ 
[34]. Finally, the levels of immune marker staining seen in our MBC samples (lower CD8, higher CD163, and higher PD-L1) is comparable to data from previous publications using TNBC samples [25, 28].

The prognosis of $\mathrm{MBC}$, when compared to TNBC, has traditionally been known to be worse, but survival outcomes have varied across numerous studies. One study examining outcomes of $\mathrm{MBC}$ and triple-negative ICNST patients found no significant differences in early DFS between the two groups [10]. A large international study comparing survival outcomes between patients with $\mathrm{MBC}$ and those with histological grade, lymph node stage, ER, and HER2 status matched conventional no specific type/invasive ductal primary breast carcinomas revealed significantly different rates of breast cancerspecific survival (BCSS-defined as the interval between primary surgery and death); however, the difference in outcomes did not remain following the exclusion of locally advanced patients [35]. Such results are similar to our multivariate analysis that showed no significant difference in OS and DDFS between the two cohorts. However, other investigations have demonstrated unfavorable results for patients with $\mathrm{MBC}$ compared to those with TNBC or hormone receptor-negative IC-NST $[6,36-38]$. The varying results highlight the need for additional larger retrospective studies to understand the biology and best treatment practices to treat patients with MBC.

We reviewed the literature to survey the chemotherapy types and regimens used in the treatment of $\mathrm{MBC}$ in comparison to TNBC and identified four studies published between 2012 and 2017, summarized in Table 5 . While survival rates for the TNBC patients in our study were similar to those in the other studies (5-year DDFS $=78.7 \%$, 5-year OS $=81.4 \%$ ), all other studies demonstrated worse prognosis for patients with $\mathrm{MBC}$ in comparison to TNBC. Notably, only one study examined the difference in the use of chemotherapy by class between $\mathrm{MBC}$ and TNBC groups [34]. While taxane therapies were used more frequently among patients with $\mathrm{MBC}$ (70.5\%) and TNBC (85.4\%) in this study compared to those in Aydiner et al. (46.3\% and $72.5 \%$, respectively), taxane use was not independently associated with either survival outcome, though our sample size was limited. Nevertheless, the use of taxane therapies might account for the relatively improved survival seen among $\mathrm{MBC}$ patients in this study compared to other cohorts of MBC patients, although this is a retrospective observation and the prospective validation of this hypothesis is difficult due to rarity of patients with MBC.

Few studies have investigated immune-related marker expression in metaplastic and triple-negative breast cancers. One study demonstrated tumor cell PD-L1 expression in $32 \%$ of $\mathrm{TNBC}$ primaries and $40 \%$ of $\mathrm{MBC}$ primaries, though the expression was rarely strong and there were only five MBC samples. In addition, PD-L1 expression within the tumor-associated inflammatory cells was seen in $61.4 \%$ of TNBC primaries, and expression was maintained between $94 \%$ of matched primary-metastatic pairs [39]. Another study further demonstrated that PDL1 expression was greater in MBC samples compared to TNBC ( $46 \%$ to $9 \%, p<0.001)$ [19]. While our study showed similar results, Joneja et al. used a greater number of $\mathrm{MBC}$ samples to establish a better match between groups. There are no published studies that compared CD8 or CD163 expression in MBC to TNBC.

CD163 is a high-affinity scavenger receptor on monocytes and macrophages that binds to hemoglobinhaptoglobin complex and innate immunity sensor of bacteria [40]. It is regulated by pro-inflammatory and anti-inflammatory mediators and plays a role in many inflammatory diseases [41]. Elevated CD163 expression has been associated with lower survival rates among various cancers [42], including breast cancer [28, 43]. Another study showed that $\mathrm{CD}_{163}{ }^{+}$macrophages in tumor stroma were positively correlated with certain pathologic characteristics seen in MBCs, such as higher grade, larger tumor size, and triple-negative/basal-like breast cancer [27]. While our study did not test for an association between CD163 expression and patient survival, our expression results show a numerical trend towards CD163 higher expression in MBC and are hypothesisgenerating. These results can be further tested in future studies with larger patient numbers.

Unlike the expression of PD-L1 and CD163, $\mathrm{CD}^{+} \mathrm{T}$ cells among tumor and stromal cells in TNBC are associated with a better prognosis and reduced risk of death [44]. Another study examined TNBC patients with high levels of $\mathrm{CD}^{+}$TILs and determined that greater levels of $\mathrm{CD}^{+}$TILs reduce the risk of recurrence and death [25]. Our study demonstrated reduced CD8 expression on immune cells in MBC samples $(p=0.02)$ in comparison to TNBC samples. These results suggest that future studies of immunotherapies for MBC could target tumor cell PD-L1, stromal CD163 ${ }^{+}$TAMs, or aim to increase the percentage of $\mathrm{CD}^{+}$immune cells. Future research could identify whether these markers act in a concerted manner to orchestrate immune suppression and if there is involvement from additional components of the tumor microenvironment.

The primary limitations of our study include its retrospective nature and the inclusion of a relatively small number of patients $(n=44)$, although this is consistent with sample sizes reported in previous studies, given the rarity of MBCs (see Table 5). As a result, more cases with longer follow-up could aid in improving the internal validity of this study. Additionally, our patient population is from only one large institution and 


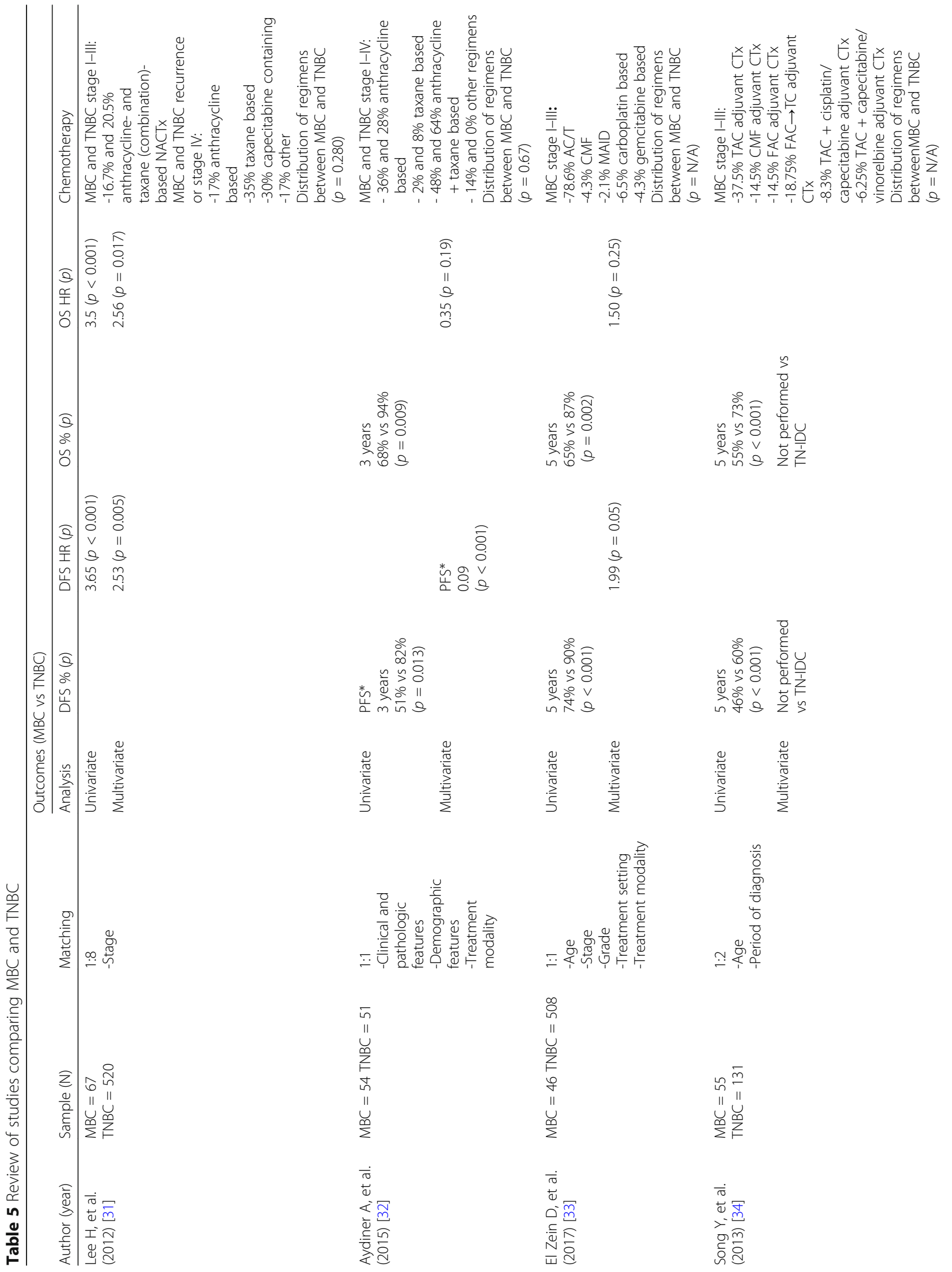


Morgan et al. World Journal of Surgical Oncology

(2020) 18:11

Page 10 of 12

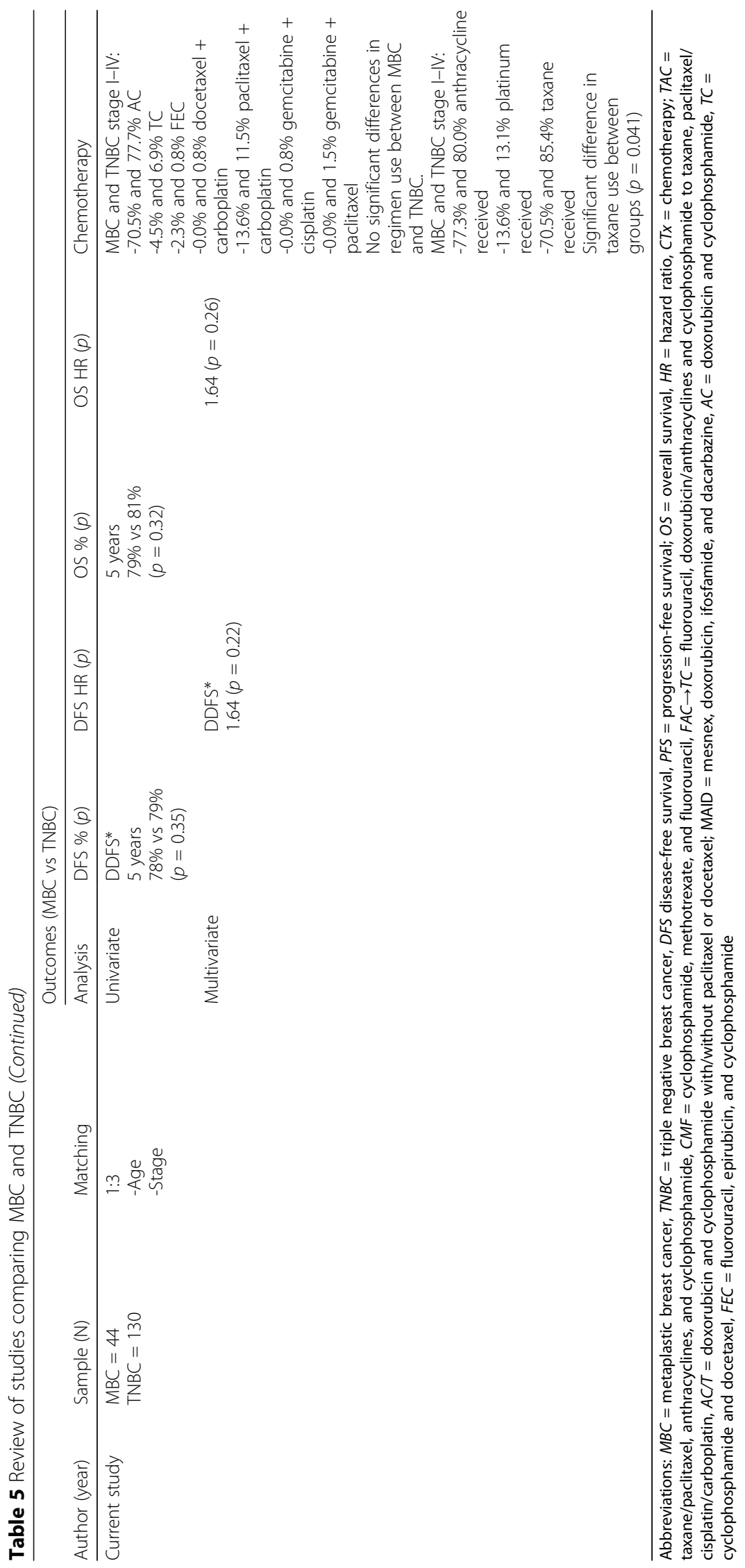


predominantly white and non-Hispanic, which is not entirely reflective of the general population. Finally, the histologic types of $\mathrm{MBC}$ and TNBC samples were not identified for reasons including a lack of availability of tissue on all clinical samples.

\section{Conclusion}

In summary, our data suggests that patients with $\mathrm{MBC}$ had similar outcomes to those with TNBC based on DDFS and OS. The use of taxane and anthracycline therapies was more common among our patients with $\mathrm{MBC}$ in comparison to another study examining this use among patients with MBC [34], although this was not independently associated with survival outcomes. Lower CD8, higher CD163, and higher PD-L1 staining in our $\mathrm{MBC}$ samples is comparable to data from previous publications using TNBC samples [25, 28]. Future studies are needed to confirm the prognostic role of tumor PDL1, stromal CD163, and tumor CD8 in MBC, and further research is needed to see if these are potential therapeutic targets. MBC is a rare disease with a small patient population, so accrual to a prospective study remains a challenge for future studies to overcome.

\begin{abstract}
Abbreviations
CD163: Cluster of differentiation 163 protein; CD8: Cluster of differentiation 8 protein; CD8 ${ }^{+}$: Cytotoxic T cell; DDFS: Distant disease-free survival; DFS: Disease-free survival; ER: Estrogen receptor; HER2: Human epidermal growth factor receptor 2; HR: Hazard ratio.; IC-NST: Invasive carcinoma of no special type; M2: Alternatively activated macrophage; MBC: Metaplastic breast cancer; MRQ26: Ventana CD8 mouse monoclonal antibody; OS: Overall survival; OSU: Ohio State University; OSUCCC-James: The Ohio State University Comprehensive Cancer Center-Arthur G. James Cancer Hospital and Richard J. Solove Research Institute; PD-1: Programmed cell death protein 1; PD-L1: Programmed death-ligand 1; PR: Progesterone receptor; SP263: Ventana PD-L1 rabbit monoclonal primary antibody; SP57: Ventana CD8 rabbit monoclonal primary antibody; TAM: Tumor-associated macrophage; TME: Tumor microenvironment; TNBC: Triple-negative breast cancer; WHO: World Health Organization; ZL: Zaibo Li, MD, PhD
\end{abstract}

\section{Acknowledgements}

The project described was supported by the Stefanie Spielman Fund for Breast Cancer Research.

\section{Authors' contributions}

Category 1: EM, AS, AG, DGS, RW, SS, AN, RR, JV, NW, MC, ZL, GY, MP, JS, JL, $A L, B R$, and $M L$ contributed to the conception and design. EM, AS, AG, GY, $J \mathrm{~L}, \mathrm{AL}, \mathrm{BR}$, and $\mathrm{ML}$ contributed to the collection and assembly of data. EM, $A S, A G, D G S, R W, Z L, G Y, M P, J S, B R$, and ML contributed to the data analysis and interpretation. Manuscript writing: EM, AS, AG, DGS, RW, SS, AN, RR, JV, $N W, M C, Z L, G Y, M P, J S, J L, A L, B R$, and $M L$ wrote the manuscript. EM, AS, AG, $D G S, R W, S S, A N, R R, J V, N W, M C, Z L, G Y, M P, J S, J L, A L, B R$, and $M L$ gave final approval of the manuscript and agreed to be accountable for all aspects of work. All authors read and approved the final manuscript.

\section{Authors' information}

Not applicable

\section{Funding}

The project described was supported by the Stefanie Spielman Fund for Breast Cancer Research and by the Award Number Grant UL1TR002733 from the National Center for Advancing Translational Sciences. The content is solely the responsibility of the authors and does not necessarily represent the official views of the National Center for Advancing Translational Sciences or the National Institutes of Health.

\section{Availability of data and materials}

The datasets used and/or analyzed during the current study are available from the corresponding author on reasonable request.

\section{Ethics approval and consent to participate}

This retrospective study meets ethical standards of the Ohio State University Comprehensive Cancer Center (OSUCCC) Clinical Scientific Review Committee (CSRC) and the Ohio State Cancer IRB, as is recognized by its approval number 2015C0135. A full waiver of HIPAA authorization has been obtained from the Ohio State Cancer IRB as this is a retrospective chart review.

\section{Consent for publication}

Not applicable. Full waiver of HIPAA authorization has been obtained from the Ohio State Cancer IRB as this is a retrospective chart review.

\section{Competing interests}

The authors declare that they have no competing interests.

\section{Author details}

${ }^{1}$ Stefanie Spielman Comprehensive Breast Cancer, The Ohio State University, Columbus, OH, USA. ${ }^{2}$ Division of Medical Oncology, Comprehensive Cancer Center, The Ohio State University Medical Center, Columbus, OH, USA. ${ }^{3}$ Department of Internal Medicine, Riverside Methodist Hospital, Columbus, Ohio, USA. ${ }^{4}$ Department of Pathology, The Ohio State University Medical Center, Columbus, OH, USA. ${ }^{5}$ Center for Biostatistics, Department of Biomedical Informatics, The Ohio State University, Columbus, OH, USA.

Received: 29 July 2019 Accepted: 29 December 2019

Published online: 14 January 2020

\section{References}

1. Greenberg D, McIntyre H, Bierre T. Metaplastic breast cancer. Australas Radiol. 2004;48:243-7 https://doi.org/10.1111/j.1440-1673.2004.01305.x.

2. Hasdemir OA, Tokgöz S, Köybaşıoğlu F, Karabacak H, Yücesoy C, Imamoğlu Gi. Clinicopathological features of metaplastic breast carcinoma. Adv Clin Exp Med. 2018; https://doi.org/10.17219/acem/68293.

3. Lakhani SR, International Agency for Research on Cancer, Weltgesundheitsorganisation, editors. WHO classification of tumours of the breast: views of a working group that convened for a consensus and editorial meeting at the International Agency for Research on Cancer (IARC) Lyon, September 1 - 3, 2011. 4. ed. Lyon: Internat. Agency for Research on Cancer; 2012.

4. Reis-Filho JS, Lakhani SR, Gobbi H, Sneige N. Metaplastic carcinoma: World Health Organization Classification of Tumours of the Breast: 2012. p. 48-52.

5. Reis-Filho JS, Milanezi F, Steele D, Savage K, Simpson PT, Nesland JM, et al. Metaplastic breast carcinomas are basal-like tumours. Histopathology. 2006; 49:10-21 https://doi.org/10.1111/j.1365-2559.2006.02467.X.

6. Jung S-Y, Kim HY, Nam B-H, Min SY, Lee SJ, Park C, et al. Worse prognosis of metaplastic breast cancer patients than other patients with triple-negative breast cancer. Breast Cancer Res Treat. 2010;120:627-37 https://doi.org/10. 1007/s10549-010-0780-8

7. Lim K-H, Oh D-Y, Chie EK, Han W, Im S-A, Kim T-Y, et al. Metaplastic breast carcinoma: clinicopathologic features and prognostic value of triple negativity. Jpn J Clin Oncol. 2010;40:112-8 https://doi.org/10.1093/ jjco/hyp139.

8. Tavassoli FA. Classification of metaplastic carcinomas of the breast. Pathol Annu. 1992;27(Pt 2):89-119.

9. Rayson D, Adjei AA, Suman VJ, Wold LE, Ingle JN. Metaplastic breast cancer: prognosis and response to systemic therapy. Ann Oncol. 1999;10:413-9.

10. Bae SY, Lee SK, Koo MY, Hur SM, Choi M-Y, Cho DH, et al. The prognoses of metaplastic breast cancer patients compared to those of triple-negative breast cancer patients. Breast Cancer Res Treat. 2011;126:471-8 https://doi. org/10.1007/s10549-011-1359-8.

11. Pezzi CM, Patel-Parekh L, Cole K, Franko J, Klimberg VS, Bland K Characteristics and treatment of metaplastic breast cancer: analysis of 892 cases from the National Cancer Data Base. Ann Surg Oncol. 2007;14:166-73 https://doi.org/10.1245/s10434-006-9124-7. 
12. Hennessy BT, Giordano S, Broglio K, Duan Z, Trent J, Buchholz TA, et al. Biphasic metaplastic sarcomatoid carcinoma of the breast. Ann Oncol. 2006; 17:605-13 https://doi.org/10.1093/annonc/mdl006.

13. Terando AM, Agnese DM, Holmes DR. Treatment and Prognosis of Rare Breast Cancers. Ann Surg Oncol. 2015;22:3225-9 https://doi.org/10.1245/ s10434-015-4748-0.

14. Stagg J, Allard B. Immunotherapeutic approaches in triple-negative breast cancer: latest research and clinical prospects. Ther Adv Med Oncol. 2013;5: 169-81 https://doi.org/10.1177/1758834012475152.

15. Smyth MJ, Ngiow SF, Ribas A, Teng MWL. Combination cancer immunotherapies tailored to the tumour microenvironment. Nat Rev Clin Oncol. 2016;13:143-58 https://doi.org/10.1038/nrclinonc.2015.209.

16. Li Z, Qiu Y, Lu W, Jiang Y, Wang J. Immunotherapeutic interventions of Triple Negative Breast Cancer. J Transl Med. 2018;16:147 https://doi.org/10. 1186/s12967-018-1514-7.

17. Pardoll DM. The blockade of immune checkpoints in cancer immunotherapy Nat Rev Cancer. 2012;12:252-64 https://doi.org/10.1038/nrc3239.

18. Teng MWL, Ngiow SF, Ribas A, Smyth MJ. Classifying Cancers Based on Tcell Infiltration and PD-L1. Cancer Res. 2015;75:2139-45 https://doi.org/10. 1158/0008-5472.CAN-15-0255.

19. Joneja U, Vranic S, Swensen J, Feldman R, Chen W, Kimbrough J, et al. Comprehensive profiling of metaplastic breast carcinomas reveals frequent overexpression of programmed death-ligand 1. J Clin Pathol. 2017;70:255-9 https://doi.org/10.1136/jclinpath-2016-203874.

20. Mittendorf EA, Philips AV, Meric-Bernstam F, Qiao N, Wu Y, Harrington S, et al. PD-L1 expression in triple-negative breast cancer. Cancer Immunol Res. 2014;2:361-70 https://doi.org/10.1158/2326-6066.CIR-13-0127.

21. Adams S, Loi S, Toppmeyer D, Cescon D, De Laurentiis M, Nanda R, et al. KEYNOTE-086 cohort B: pembrolizumab monotherapy for PD-L1--positive, previously untreated, metastatic triple-negative breast cancer (mTNBC). Cancer Res. 2018.

22. Emens LA, Cruz C, Eder JP, Braiteh F, Chung C, Tolaney SM, et al. Long-term Clinical Outcomes and Biomarker Analyses of Atezolizumab Therapy for Patients With Metastatic Triple-Negative Breast Cancer: A Phase 1 Study. JAMA Oncol. 2018; https://doi.org/10.1001/jamaoncol.2018.4224.

23. Adams S. Dramatic response of metaplastic breast cancer to chemoimmunotherapy. NPJ Breast Cancer. 2017;3:8 https://doi.org/10.1038/s41523017-0011-0.

24. Tolaney SM, Kalinsky K, Kaklamani V, Savulsky C, Olivo M, Aktan G, et al. Abstract PD6-13: Phase 1b/2 study to evaluate eribulin mesylate in combination with pembrolizumab in patients with metastatic triplenegative breast cancer. Cancer Res. 2018;78:PD6-13 https://doi.org/10.1158/ 1538-7445.SABCS17-PD6-13.

25. Miyashita M, Sasano H, Tamaki K, Hirakawa H, Takahashi Y, Nakagawa S, et al. Prognostic significance of tumor-infiltrating CD8+ and FOXP3+ lymphocytes in residual tumors and alterations in these parameters after neoadjuvant chemotherapy in triple-negative breast cancer: a retrospective multicenter study. Breast Cancer Res. 2015;17:124.

26. Barros MHM, Hauck F, Dreyer JH, Kempkes B, Niedobitek G. Macrophage polarisation: an immunohistochemical approach for identifying $\mathrm{M} 1$ and $\mathrm{M} 2$ macrophages. PLoS One. 2013;8:e80908 https://doi.org/10.1371/journal. pone.0080908

27. Medrek C, Pontén F, Jirström K, Leandersson K. The presence of tumor associated macrophages in tumor stroma as a prognostic marker for breast cancer patients. BMC Cancer. 2012;12:306 https://doi.org/10.1186/1471-2407-12-306.

28. Adams TA, Vail PJ, Ruiz A, Mollaee M, McCue PA, Knudsen ES, et al. Composite analysis of immunological and metabolic markers defines novel subtypes of triple negative breast cancer. Mod Pathol. 2018;31:288-98 https://doi.org/10.1038/modpathol.2017.126.

29. Hu H, Hang J-J, Han T, Zhuo M, Jiao F, Wang L-W. The M2 phenotype of tumor-associated macrophages in the stroma confers a poor prognosis in pancreatic cancer. Tumour Biol. 2016;37:8657-64 https://doi.org/10.1007/ s13277-015-4741-z.

30. Bergstralh EJ, Kosanke JL, Jacobsen SJ. Software for optimal matching in observational studies. Epidemiology. 1996;7:331-2.

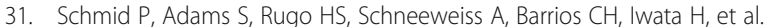
Atezolizumab and Nab-Paclitaxel in Advanced Triple-Negative Breast Cancer. N Engl J Med. 2018;379:2108-21 https://doi.org/10.1056/NEJMoa1809615.

32. Hou Y, Nitta H, Wei L, Banks PM, Parwani AV, Li Z. Evaluation of Immune Reaction and PD-L1 Expression Using Multiplex Immunohistochemistry in HER2-Positive Breast Cancer: The Association With Response to Anti-HER2
Neoadjuvant Therapy. Clin Breast Cancer. 2018;18:e237-44 https://doi.org/ 10.1016/j.clbc.2017.11.001.

33. Lee H, Jung S-Y, Ro JY, Kwon Y, Sohn JH, Park IH, et al. Metaplastic breast cancer: clinicopathological features and its prognosis. J Clin Pathol. 2012;65: 441-6 https://doi.org/10.1136/jclinpath-2011-200586.

34. Aydiner A, Sen F, Tambas M, Ciftci R, Eralp Y, Saip P, et al. Metaplastic breast carcinoma versus triple-negative breast cancer: Survival and Response to Treatment. Medicine. 2015;94:e2341 https:/doi.org/10.1097/MD.0000000000002341.

35. Rakha EA, Tan PH, Varga Z, Tse GM, Shaaban AM, Climent F, et al. Prognostic factors in metaplastic carcinoma of the breast: a multi-institutional study. $\mathrm{Br}$ J Cancer. 2015;112:283-9 https://doi.org/10.1038/bjc.2014.592.

36. El Zein D, Hughes M, Kumar S, Peng X, Oyasiji T, Jabbour H, et al. Metaplastic carcinoma of the breast is more aggressive than triple-negative breast cancer: a study from a single institution and review of literature. Clin Breast Cancer. 2017;17:382-91 https://doi.org/10.1016/j.clbc.2017.04.009.

37. Song $Y$, Liu $X$, Zhang $G$, Song $H$, Ren $Y$, He X, et al. Unique clinicopathological features of metaplastic breast carcinoma compared with invasive ductal carcinoma and poor prognostic indicators. World J Surg Oncol. 2013;11:129 https://doi.org/10.1186/1477-7819-11-129.

38. Nelson RA, Guye ML, Luu T, Lai LL. Survival outcomes of metaplastic breast cancer patients: results from a US population-based analysis. Ann Surg Oncol. 2015:22:24-31 https://doi.org/10.1245/s10434-014-3890-4.

39. Dill EA, Gru AA, Atkins KA, Friedman LA, Moore ME, Bullock TN, et al. PD-L1 Expression and Intratumoral Heterogeneity Across Breast Cancer Subtypes and Stages: An Assessment of 245 Primary and 40 Metastatic Tumors. Am J Surg Pathol. 2017:41:334-42 https://doi.org/10.1097/PAS.0000000000000780.

40. Van Gorp H, Delputte PL, Nauwynck HJ. Scavenger receptor CD163, a Jackof-all-trades and potential target for cell-directed therapy. Mol Immunol. 2010;47:1650-60 https://doi.org/10.1016/j.molimm.2010.02.008.

41. Buechler C, Ritter M, Orsó E, Langmann T, Klucken J, Schmitz G. Regulation of scavenger receptor CD163 expression in human monocytes and macrophages by pro-and antiinflammatory stimuli. J Leukoc Biol. 2000;67:97-103.

42. Jung KY, Cho SW, Kim YA, Kim D, Oh B-C, Park DJ, et al. Cancers with Higher Density of Tumor-Associated Macrophages Were Associated with Poor Survival Rates. J Pathol Transl Med. 2015;49:318-24 https://doi.org/10.4132/ jptm.2015.06.01.

43. Hollmén M, Karaman S, Schwager S, Lisibach A, Christiansen AJ, Maksimow $M$, et al. G-CSF regulates macrophage phenotype and associates with poor overall survival in human triple-negative breast cancer. Oncoimmunology. 2016;5:e1115177 https://doi.org/10.1080/2162402X.2015.1115177.

44 Ali HR, Provenzano E, Dawson S-J, Blows FM, Liu B, Shah M, et al. Association between CD8+ T-cell infiltration and breast cancer survival in 12,439 patients. Ann Oncol 2014;25:1536-43 https://doi.org/10.1093/ annonc/mdu191.

\section{Publisher's Note}

Springer Nature remains neutral with regard to jurisdictional claims in published maps and institutional affiliations.
Ready to submit your research? Choose BMC and benefit from:

- fast, convenient online submission

- thorough peer review by experienced researchers in your field

- rapid publication on acceptance

- support for research data, including large and complex data types

- gold Open Access which fosters wider collaboration and increased citations

- maximum visibility for your research: over $100 \mathrm{M}$ website views per year

At $\mathrm{BMC}$, research is always in progress.

Learn more biomedcentral.com/submission 